“ (C) 2013 IEEE. Personal use of this material is permitted. Permission from IEEE must be obtained for all other uses, in any current or future media, including

reprinting/republishing this material for advertising or promotional purposes, creating new collective works, for resale or redistribution to servers or lists, or reuse of any copyrighted component of this work in other works." 


\title{
The Advancement of an Obstacle Avoidance Bayesian Neural Network for an Intelligent Wheelchair
}

\author{
Anh V. Nguyen, Lien B. Nguyen, Steven Su, Member, IEEE, and Hung T. Nguyen, \\ Senior Member, IEEE
}

\begin{abstract}
In this paper, an advanced obstacle avoidance system is developed for an intelligent wheelchair designed to support people with mobility impairments who also have visual, upper limb, or cognitive impairment. To avoid obstacles, immediate environment information is continuously updated with range data sampled by an on-board laser range finder URG-04LX. Then, the data is transformed to find the relevant information to the navigating process before being presented to a trained obstacle avoidance neural network which is optimized under the supervision of a Bayesian framework to find its structure and weight values. The experiment results showed that this method allows the wheelchair to avoid collisions while simultaneously navigating through an unknown environment in real-time. More importantly, this new approach significantly enhances the performance of the system to pass narrow openings such as door passing.
\end{abstract}

\section{INTRODUCTION}

Commercial electric - powered wheelchairs traced to the 1950s have been providing functional mobility for people with both lower and upper extremity impairments. These include various overlapping motor, perceptual, or cognitive impairments such as spinal cord injury, or cerebral palsy. With the assistance of the wheelchair, their quality of life is significantly improved [1]. Although the benefits of the powered wheelchair are well-documented, safety issues and difficulties associated with the operation and control of power wheelchairs often prevent clinicians and rehabilitation professionals from prescribing powered mobility [2]. An intelligent wheelchair has been, therefore, studied to accommodate these people. According to the report [3], 61\% to $91 \%$ of all wheelchair users in the US would benefit from the assistance of an intelligent wheelchair.

One of the indispensable functions for an intelligent wheelchair system is an automatic obstacle avoidance function. This function is necessary to provide safe operations for the operator, normally elderly or disabled people, in unknown environments, because a human operated wheelchair system is expected to work in various places such as house or hospital rooms. For this reason, only real-time collision avoidance methods are applicable to this application. Besides, considered as an assistive system for humans, obstacle avoidance should result in smooth trajectories and comfort for the user during daily driving manoeuvres.

Anh V. Nguyen, Lien B. Nguyen, Steven Su, Hung T. Nguyen are with Faculty of Engineering and Information Technology, University of Technology, Sydney, Broadway, NSW 2007, Australia, (e-mail: Anh.Nguyen-3@student.uts.edu.au; BichLien.Nguyen@student.uts.edu.au; Steven.Su@uts.edu.au; Hung.Nguyen@uts.edu.au ).

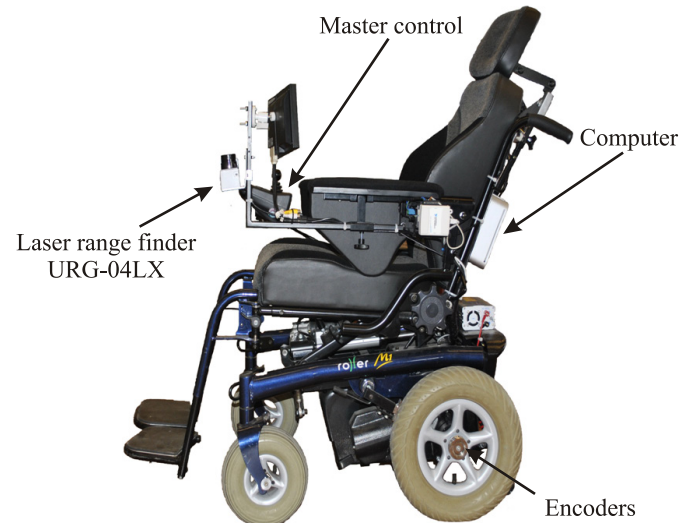

Fig.1. A prototype of intelligent wheelchair at Centre for Health Technologies, University of Technology, Sydney.

Although a variety of obstacle avoidance methods for intelligent wheelchairs have been reported, they can be briefly divided into two kinds. The first paradigm is known as route programs using step by step code for instructing the wheelchair. Methods that have been refined over the years and are still useful and popular such as Potential field [4], Vector field histogram $[5,6]$, or rule-based approaches $[7,8]$ are typically classified as this type. Obviously, these approaches are implemented because they are explicit and rigorous. But, it is sometimes very hard to describe the desired, exact wheelchair' behavior in some practical situations by proper formulas or code statements.

The other paradigm that replaces explicit programming by training the machine to avoid obstacles, as the wheelchair moves around in a cluttered environment, has been emerging in recent years. Instead of using formulas or code statements, the wheelchair first learns how to adapt to certain situations, and after learning, the wheelchair then is able to move without collisions. Methods [9] are feasible to transfer human navigation skills to an intelligent wheelchair, but only applied in simulation or static environment. In recent years, obstacle avoidance methods based on Bayesian neural networks $[10,11]$ developed for our intelligent wheelchair called SAM (semi-autonomous machine, see Fig.1) - enable the wheelchair to have collision - free navigation in real time in a dynamic environment.

Although demonstrated to work well in various dynamic environments, the wheelchair with obstacle avoidance neural network developed in our previous work [11] sometimes has difficulty in passing through the doorways of the width which is less than $1 \mathrm{~m}$. In order to overcome the difficulty, in this paper, rather than using raw data [11], the data is transformed by considering how surrounding obstacles effect the navigating process to extract relevant information before 
presented to the network. Experiment results show that the new proposed method increases the generalization and efficiency of the trained network which helps the wheelchair not only effectively avoid obstacles while navigating to the local target, but also considerably enhance the door passing performance of the system. This paper is organized as follows. In Section II, the method will be presented. In section III, experimental results of the proposed method are described to demonstrate the performance of the assistive navigation system. Finally, a conclusion of our study is drawn in Section IV.

\section{METHOD}

\section{A. System}

Our SAM, as shown Fig.1, based on a commercial wheelchair which has been modified to attach main additional items: a minicomputer, user interfaces, and measurement sensors. This modification allows the user to operate in both manual mode and assistive mode. In the manual mode, SAM acts as a normal powered wheelchair in which the operator simply uses a joystick to drive the wheelchair. In assistive mode operation, the system interrupts the connection between the wheelchair joystick and wheel motor controllers. By doing so, the on-board computer generates control signals sent to the motor controllers following calculations of a navigation algorithm relying on user intentions and measured surrounding obstacles. To control the system, the user can use one of four available interface types compatible to SAM: Joystick, head movement, Brain computer interface, iPhone4/iPad. SAM automatically detects and reads signals whenever they are connected to the system. Through these user interface signals, the SAM is able to monitor what the user wants it to do. Another important information source used to make navigation decisions comes from the laser URG-04LX-based obstacle detection. The computer triggers the sensor to produce measured distance and angle data of surrounding obstacles with maximum radius of $4 \mathrm{~m}$ and angular resolution of $1.08^{0}$. Each scan time, 180 data points corresponding to front obstacles are obtained.

When assistive mode is active, SAM starts to collect data from both the user interface and sensors at $100 \mathrm{~ms}$ sampling rate. Then, SAM combines these data together to find the direction of travel where the user intends to go. While one of its requirements is to avoid obstacles, and provide a smooth trajectory, a trained collision avoidance neural network is used to make final decisions about editing the direction of travel as well as the speed of the vehicle. The following sections provide detail description about this network.

\section{B. Direction of travel}

SAM uses URG-04LX data to an build on-line map and determine a number of potential paths for circumnavigation. In particular, the wheelchair calculates the size of a path opening, if the size is greater than the vehicle's width size plus a safe margin, the path is considered as one of potential paths of travel. Next, these potential paths and user's commands are combined to find the most suitable direction
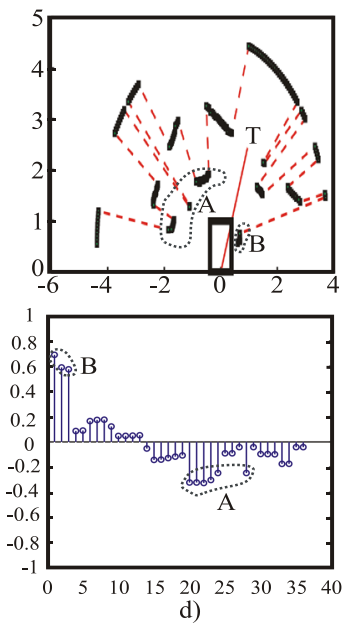

Fig.2. Steps to transform URG-04LX data b)
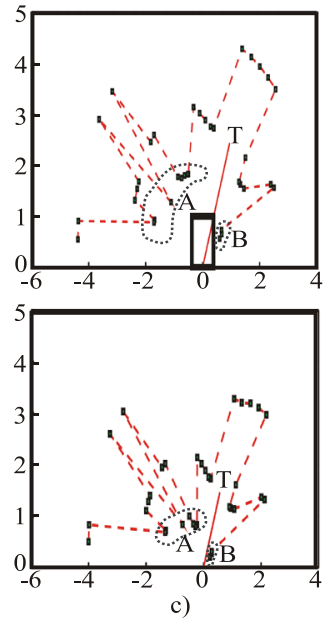

of travel or a local target by using Bayesian recursive technique presented in [12]. This technique solves the uncertainty of signals caused by the limitation of devices and inconsistent commands given by the impaired user.

\section{Environment analysis}

When the direction of travel is determined, the next task is how to get there without collision and provides a smooth trajectory. Taking into consideration the wheelchair's control in a relationship of surrounding obstacles and the target $\mathrm{T}$ as Fig. 2 a) displaying all instantaneously measured obstacle distances to the wheelchair in a polar form at a time and wheelchair dimensions, then clearly, not all of these obstacles play an equally important role in navigating the wheelchair. In other words, obstacles in the $\mathrm{A}$ and $\mathrm{B}$ regions have significant effects on both speed and steering direction of the wheelchair while others far from the wheelchair play none. Thus, it is necessary to select important environment features and represent them in appropriate forms facilitating the use for the non-collision neural network in the next section. This can be obtained through the following four steps:

Step 1: Remove unnecessary environment data points. For instance, divide the 180 URG-04LX data points into 36 sections - 5 points per each section, and the closest obstacle point of each section represents that section. By the number of tries, 5 - point section is likely to be optimal as, if the number of points in each section is chosen to be too large, some important points might lose. If the number is too small, it results in too many sections. The result of this step is displayed in the Fig. 2b).

Step 2: From chosen data from step 1, take the wheelchair's dimensions into account to find the real distance of selected obstacles to the wheelchair shown Fig. 2 c).

Step 3: Transform all values from step 3 by this normalized formula

$$
I_{n t h}=\frac{a}{\left(d_{n t h}+b\right)^{2}}
$$


Where: $I_{n t h}$ and $d_{n t h}$ are values of $n^{\text {th }}$ section after and before transforming. $\mathrm{a}, \mathrm{b}$ are constants that are determined such as $I_{\text {nth }}$ changes from 0 to 1 when $d_{\text {nth }}$ ranges $4(m)$ to $0.1(\mathrm{~m})$.

Step 4: As effects of obstacles in different positions to the target are different, a positive or negative sign is assigned for each value of section. For example, those lying on the right side of the travel direction tend to push the vehicle towards the left, resulting in the steering angle being larger than the angle to the target, so that they have positive effects. Similarly, the left side obstacles lead the steering angle to be smaller than the goal angle, and therefore have negative impacts. The results of step 3 and 4 are represented in the Fig.2 d).

\section{Neural network training based on Bayesian framework}

For an obstacle avoidance task, a feed forward neural network with sigmoid hidden neurons and linear output neurons is recruited to learn how to react. 36 transformed URG04-LX data points and a direction of travel are considered as inputs. Output layer comprises of two outputs corresponding to steering angle control and velocity control. A number of hidden nodes are determined during the training process under Bayesian supervision.

Bayesian framework has been carried out within the Levenberg - Marquardt optimization approach to find the most optimal network structure as described in detail in our previous work [11]. While training, the number of hidden nodes varies, and the assessment process for each network structure is taken as follows. At first, the values of the hyperparameters and weights' value of the network are randomly initialized, then, the Levenberg - Marquardt optimization algorithm updates the weight's value in order to minimize the total error function. Finally, the evidence value of the structure is estimated when the optimization algorithm is converged. The most suitable network is selected with the highest evidence value.

\section{EXPERIMENT RESULTS}

\section{A. Neural network training}

\section{Collecting training data experiments.}

In order to train the network to learn how to react in certain situations, acquisition experiments are implemented in various indoor environments to gather training patterns. While SAM is manually driven to follow a number of predesigned paths by a standard joystick, a software records measured obstacle distances to the wheelchair and control signals at $100 \mathrm{~ms}$ sampling rate. Each pattern includes 37 inputs and 2 outputs as described in the above section.

All acquisition experiments are carried out in the Building 1 of the University of Technology, Sydney (UTS) because it has various environment types such as a narrow space, walls, doorways, and moving obstacles. While driving the wheelchair, the user is aware that depending on obstacle clearance level and types of environment, the wheelchair is controlled differently. For example, for the experiments in the Foyer of the UTS Building 1 where the obstacles are far, the wheelchair would be driven close to the direction of travel and at a fast speed - the maximum speed is set at $0.8 \mathrm{~m} / \mathrm{s}$ in these experiments. In contrast, when moving between two closely spaced obstacles such as the posts of a doorway, the wheelchair is slowed down, and centered with the doorway - the maximum speed for passing door task is only set at $0.5 \mathrm{~m} / \mathrm{s}$. In this situation, a slight difference between a steering angle and the desired direction is usually acceptable. For corridors or rooms with long walls experiments, the speed of the wheelchair might be up to 0.8 $\mathrm{m} / \mathrm{s}$, however, more importantly, the wheelchair should be driven to run parallel to the walls.

\begin{tabular}{lr} 
Environment type & Number of Patterns \\
\hline $\begin{array}{l}\text { The Foyer of the Building 1 with general } \\
\text { obstacles such as boxes, chairs, tables, } \\
\text { and moving people }\end{array}$ & $\mathbf{3 0 0}$ \\
\hline Corridors with different width sizes & $\mathbf{2 5 0}$ \\
\hline Rooms with long walls & $\mathbf{2 5 0}$ \\
\hline Doors with varied sizes in width & $\mathbf{4 0 0}$ \\
\hline Total & $\mathbf{1 2 0 0}$ \\
\hline Tab.1. Patterns collected from the acquisition data experiments
\end{tabular}

The table 1 details a number of patterns collected from the acquisition data experiments. All patterns are utilized for training purposes.

\section{Bayesian neural network training results}

During training, a collection of feed-forward neural networks with different hidden nodes set to vary from 1 to 10 are assessed. For consistency, all steps applied for each network structure are repeated 3 times, and the results are reported in the Fig.3). The network architecture with 4 hidden nodes which yields the highest evidence is considered as the best one and selected to control the wheelchair.

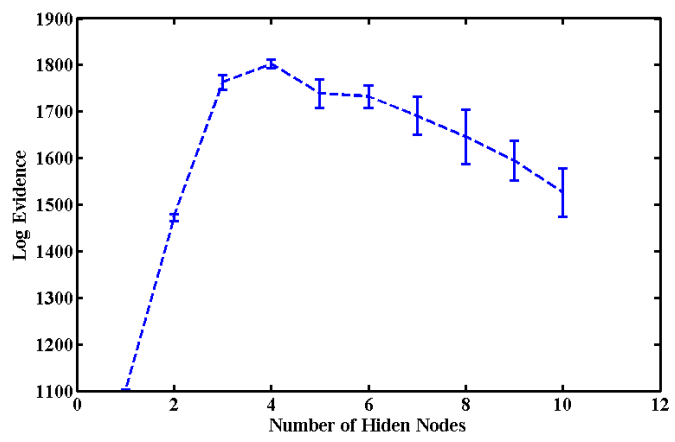

Fig.3. Log evidence estimation result for hidden nodes

\section{B. Performance valuation}

\section{Real-time navigation test}

In order to evaluate a performance of the newly designed obstacle avoidance network, SAM is asked to perform a blue dash trajectory: $\mathrm{AB}$ - general avoidance, $\mathrm{BC}$ - door passing, $\mathrm{CD}$ - wall following, DF - moving obstacle avoidance and door passing in the laboratory room Fig.4. User commands only tell it to go at start, then turn right, forward, turn right and stop through the head movement interface control. It is noticeable that this environment is unknown to the SAM to 


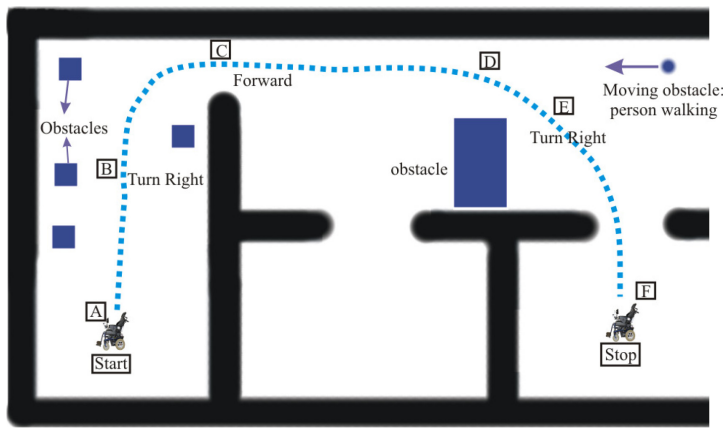

Fig.4.The automated assistive navigation system's performance

verify the generalization of the trained neural network. Five trials are administered, and the average speed per each segment and a number of collisions of each trial are recorded in table 2.

\begin{tabular}{|l|r|r|r|r|c|}
\hline & $\begin{array}{c}\text { Average speed } \\
\text { per AB }(\mathrm{m} / \mathrm{s})\end{array}$ & $\begin{array}{c}\text { Average speed } \\
\text { per BC }(\mathrm{m} / \mathrm{s})\end{array}$ & $\begin{array}{c}\text { Average speed } \\
\text { per CD }(\mathrm{m} / \mathrm{s})\end{array}$ & $\begin{array}{c}\text { Average speed } \\
\text { per DF }(\mathrm{m} / \mathrm{s})\end{array}$ & $\begin{array}{c}\text { Number of } \\
\text { collisions }\end{array}$ \\
\hline Trial 1 & 0.81 & 0.32 & 0.64 & 0.36 & 0 \\
\hline Trial 2 & 0.76 & 0.37 & 0.7 & 0.34 & 0 \\
\hline Trial 3 & 0.83 & 0.36 & 0.67 & 0.38 & 0 \\
\hline Trial 4 & 0.78 & 0.32 & 0.68 & 0.4 & 0 \\
\hline Trial 5 & 0.82 & 0.39 & 0.67 & 0.39 & 0 \\
\hline Average & 0.8 & 0.352 & 0.672 & 0.374 & \\
\hline Deviation & 0.029 & 0.031 & 0.022 & 0.024 & \\
\hline
\end{tabular}

Tab.2. The average speed and number of collision in the five trails

The result indicates that SAM is able to navigate through an unknown environment in which both static obstacles and dynamic obstacles (person walking) are present. No collisions occur during the trials. The wheelchair runs quite fast when obstacles are far $(\mathrm{AB}-0.8 \pm 0.029 \mathrm{~m} / \mathrm{s})$ or wall following $(\mathrm{CD}-0.672 \pm 0.022 \mathrm{~m} / \mathrm{s})$, while slowing down as entering narrow spaces $(\mathrm{BC}-0.352 \pm 0.031 \mathrm{~m} / \mathrm{s}$ and $\mathrm{DF}-$ $0.374 \pm 0.024 \mathrm{~m} / \mathrm{s}$ )

\section{Door passing}

Door passing experiment is conducted to test the ability of SAM to pass between closely spaced obstacles such as a door. In this experiment, a door having a changeable width from $0.75 \mathrm{~m}$ to $1.2 \mathrm{~m}$ was used. In each trial, the wheelchair $2 \mathrm{~m}$ away from the door opening is driven toward the door. Ten trials are managed for each door-width setting. The percentage of successful passing was record in table 3 .

\begin{tabular}{|c|c|}
\hline Door width (cm) & Rate of succeful passing (100\%) \\
\hline 75 & 80 \\
\hline 80 & 90 \\
\hline 90 & 100 \\
\hline 100 & 100 \\
\hline 110 & 100 \\
\hline 120 & 100 \\
\hline
\end{tabular}

Tab.3. Door passing test results

As shown in table 3, SAM is able to cross doorways of width $75 \mathrm{~cm}$ with $80 \%, 80 \mathrm{~cm}$ with $90 \%, 90 \mathrm{~cm}$ or more with $100 \%$.

\section{CONCLUSION}

The result of experiments indicate that a system based on a trained Bayesian neural network can provide a good navigation and a reliable collision avoidance system for wheelchair users who have vision, upper-limb, or cognitive disabilities; simply have difficulty mastering safe wheelchair driving skills.

The method proposed in this paper increases the generalization and efficiency of the network. In [11], the optimal non-collision network structure requires six hidden nodes. With the new method, the network only needs four hidden nodes to navigate the wheelchair to avoid obstacles. That means the computing time during navigating is shorter, resulting in the system responding faster. This might explain why the results of using both networks are quite similar for real-time navigation in the laboratory room where obstacles are relatively far or doors are quite wide (approximately $1.1 \mathrm{~m})$. However, the four hidden node network improves door passing performance of the wheelchair when SAM is able to pass the doorways of width $80 \mathrm{~cm}$ with $90 \%$ and $90 \mathrm{~cm}$ with $100 \%$, while with the six hidden node network, SAM is only able to cross those doorways with $50 \%$ and $80 \%$ respectively.

\section{REFERENCES}

[1] E. Trefler, S. G. Fitzgerald, D. A. Hobson, T. Bursick, and R. Joseph, "Outcomes of wheelchair systems intervention with residents of longterm care facilities," Assistive Technology, vol. 16, pp. 18-27, Sum 2004.

[2] D. Gavin-Dreschnack, A. Nelson, S. Fitzgerald, J. Harrow, A. SanchezAnguiano, S. Ahmed, and G. Powell-Cope, "Wheelchair-related falls Current evidence and directions for improved quality care," Journal of Nursing Care Quality, vol. 20, pp. 119-127, Apr-Jun 2005.

[3] R. C. Simpson, E. F. LoPresti, and R. A. Cooper, "How many people would benefit from a smart wheelchair?," Journal of Rehabilitation Research and Development, vol. 45, pp. 53-71, 20082008.

[4] M. R. Petry, A. P. Moreira, R. A. M. Braga, and L. P. Reis, "Shared control for obstacle avoidance in intelligent wheelchairs," in Robotics Automation and Mechatronics (RAM), 2010 IEEE Conference on, pp. 182-187.

[5] R. Kurozumi, K. Tsuji, S. i. Ito, K. Sato, S. Fujisawa, and T. Yamamoto, "Experimental validation of an online adaptive and learning obstacle avoiding support system for the electric wheelchairs," in Systems Man and Cybernetics (SMC), 2010 IEEE International Conference on, pp. 92-99.

[6] S.P.Levine, D.A. Bell, L.A. Jaros, R.C. Simpson,Y.Koren, and J. Borenstein, "The NavChair Assistive Wheelchair Navigation System," Rehabilitation Engineering, IEEE Transactions on, vol. 7, pp. 443-451, 1999.

[7] R. Simpson, E. LoPresti, S. Hayashi, I. Nourbakhsh, and D. Miller, "The Smart Wheelchair Component System," Journal of Rehabilitation Research and Development, vol. 41, pp. 429-442, May-Jun 2004.

[8] C. Galindo, J. Gonzalez, and J. A. Fernandez-Madrigal, "Control architecture for human-robot integration: Application to a robotic wheelchair," Ieee Transactions on Systems Man and Cybernetics Part B-Cybernetics, vol. 36, pp. 1053-1067, Oct 2006.

[9] H. N. Chow and X. Yangsheng, "Learning Human Navigational Skil for Smart Wheelchair in a Static Cluttered Route," Industrial Electronics, IEEE Transactions on, vol. 53, pp. 1350-1361, 2006.

[10]H. T. Trieu, H. T. Nguyen, and K. Willey, "Obstacle avoidance for power wheelchair using Bayesian neural network," in Annual International Conference of the Ieee Engineering in Medicine and Biology Society, Vols 1-16, 2007., 2007, pp. 4771-4774.

[11]A. V.Nguyen., L. Nguyen, S. Su., and H. T.Nguyen., "Development of a Bayesian Neural Networks to Perform Obstacle Avoidance for an Intelligent Wheelchair," in 34 rd Annual International Conference of the IEEE EMBS., San Diego, California, USA, 2012.

[12]A. V.Nguyen., S. Su., and H. T.Nguyen., "Development of a Bayesian Recursive Algorithm to Find Free-Spaces for an Intelligent Wheelchair.," in 33 rd Annual International Conference of the IEEE EMBS., Boston, Massachusetts USA, 2011. 